\title{
Homenaje a Irmgard Weitlaner Johnson (1914-2011)
}

Alejandro de Ávila Blomberg

\section{CpenEdition}

Journals

Edición electrónica

URL: https://journals.openedition.org/jsa/11992

DOI: 10.4000/jsa.11992

ISSN: 1957-7842

Editor

Société des américanistes

Edición impresa

Fecha de publicación: 20 diciembre 2011

Paginación: 383-387

ISSN: 0037-9174

\section{Referencia electrónica}

Alejandro de Ávila Blomberg, «Homenaje a Irmgard Weitlaner Johnson (1914-2011)», Journal de la Société des américanistes [En línea], 97-2 | 2011, Publicado el 22 enero 2012, consultado el 04 septiembre 2022. URL: http://journals.openedition.org/jsa/11992 ; DOI: https://doi.org/10.4000/jsa. 11992

All rights reserved 


\section{NÉCROLOGIES}

\section{Homenaje a Irmgard WEITLANER JOHNSON}

(1914-2011)

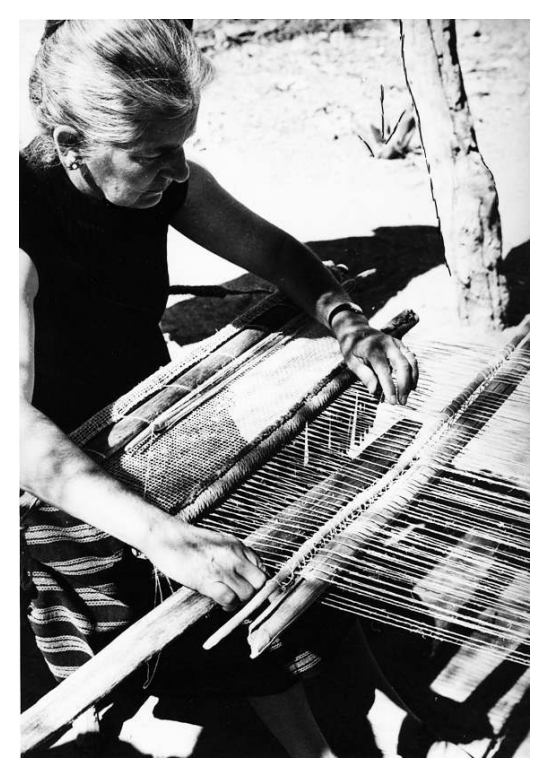

La obra de la maestra Irmgard Weitlaner Johnson representa la investigación de mayor rigor y profundidad sobre uno de los legados culturales más importantes de nuestro país. A lo largo de setenta años de trabajo, la maestra Johnson dedicó su vida al textil indígena. Enfocándose tanto en los aspectos técnicos del tejido como en su contexto etnográfico, y abordando la memoria histórica que constituyen sus diseños, la obra de la maestra Johnson nos ha permitido apreciar al textil como una de las expresiones más diversificadas, más sofisticadas en su ejecución y más significativas en su iconografía, de la cultura de México.

Sus trabajos publicados marcan seis décadas y cubren todos los tejidos sobresalientes, incluyendo tanto los textiles arqueológicos más importantes descubiertos en el siglo xx como las piezas coloniales, decimonónicas y contemporáneas más interesantes. La maestra Johnson colaboró en diversas investigaciones arqueológicas, analizando los restos hechos con fibras. Entre otros proyectos, participó con el Dr. Richard MacNeish en el estudio de los importantes hallazgos de la Cueva de Coxcatlán y otros sitios del Valle de 
Tehuacán, describiendo y publicando las evidencias más tempranas del tejido en Mesoamérica. Colaboró también con el Dr. Alfonso Caso en el estudio de documentos coloniales tempranos pintados sobre tela, como el Lienzo de Ocotepec, pueblo mixteco de Oaxaca. Analizó y dio a conocer los textiles arqueológicos de Chiptic en Chiapas, que mostraron técnicas que no habían sido registradas antes en México. Su libro sobre la Cueva de la Candelaria en Coahuila, publicado por el Instituto Nacional de Antropología e Historia, es hasta la fecha el estudio más completo y detallado sobre tejidos arqueológicos de nuestro país.

En la literatura técnica sobre el textil, la obra de la maestra Johnson ha merecido reconocimiento internacional. Su tesis de maestría sobre los enlazados de urdimbre sin trama conocidos como sprang, abarcando ejemplos de todo el continente, es la referencia más importante sobre el tema y es citada ampliamente en los trabajos de Emery (1966) y Seiler-Baldinger (1994), que son considerados como los textos clásicos sobre las técnicas del tejido a nivel mundial. El estudio de la maestra Johnson sobre el tejido de trama envolvente en una pieza arqueológica de Durango y en los huipiles de Choapan en Oaxaca fue particularmente trascendente al describir una estructura de tejido que aparentemente se inventó en México, y que no se encuentra más al sur. Este trabajo demostró la sofisticación técnica y la creatividad de las antiguas artistas mexicanas del telar, frente al sesgo de algunos autores norteamericanos y europeos por considerar al área andina como el centro de origen de toda la tecnología textil en el continente, visualizando a las tejedoras mesoamericanas como receptoras pasivas de las innovaciones técnicas difundidas desde el sur.

En sus investigaciones etnográficas, la maestra Johnson definió los estándares para el estudio y la conservación del textil indígena. Su labor se desarrolló en una época en la que la tendencia general en los medios de comunicación, e inclusive en publicaciones académicas, era folclorizar la indumentaria tradicional como «trajes regionales ». Grandes colecciones de textiles fueron formadas y exhibidas sin registrar siquiera el origen o el uso tradicional de las piezas, « embelleciendo » en muchos casos las prendas mediante decoraciones postizas. El rigor antropológico y el respeto hacia las culturas indígenas con que la maestra Johnson documentó los procesos de producción y el uso de los tejidos fue determinante en la formación de las colecciones etnográficas del Museo Nacional de Artes e Industrias Populares (acervo que resguarda actualmente la Comisión para el Desarrollo de los Pueblos Indígenas), primero, y el Museo Nacional de Antropología después. Ella colaboró con ambos museos por largo tiempo, y un número importante de las piezas y registros fotográficos que conservan son resultado de sus investigaciones de campo. Asesoró también a museógrafos y conservadores relacionados con el textil en otros museos dentro y fuera del país.

Sus archivos, biblioteca y colección personal de tejidos constituyen indudablemente el acervo particular más importante sobre textiles mexicanos. Incluyen 
un registro fotográfico de primer orden, que comprende negativos tomados entre los años 1930 y 1960 de técnicas y estilos del tejido que han desaparecido o se han transformado radicalmente en las últimas décadas. En este sentido, la obra de la maestra Johnson es irreemplazable: sus notas de campo, fotografías y muestras de textiles son testimonios del proceso de cambio más acelerado en la historia del tejido en México, a raíz de la profunda transformación social y económica de las áreas rurales del país al mediar el siglo pasado. En una época en que la opinión general, aun entre intelectuales, tendía a desvalorar las colecciones etnográficas como objetos fácilmente reemplazables, visualizando a las culturas indígenas como inmutables, ella enfatizó la importancia de documentar y conservar de manera sistemática muestras representativas de diferentes técnicas y estilos del arte popular, previendo cambios inminentes.

Los trabajos de la maestra Johnson son punto de partida para toda investigación sobre el textil en México. Sus numerosas publicaciones sobre tejidos contemporáneos son citadas ampliamente en la literatura etnográfica. Podemos destacar entre ellas el detallado estudio sobre los tejidos de telar de cintura de Tuxpan, Jalisco, una tradición que ha desaparecido por completo, y que sería prácticamente desconocida de no ser por esa investigación; los trabajos sobre tejidos coloniales y contemporáneos decorados con plumas, de particular interés estético, línea a la que damos continuidad en este museo; las investigaciones sobre el juishu otomí de Temoaya, una prenda única en Mesoamérica casi olvidada, y sobre los enredos zapotecos de Mitla, que exhiben una técnica compleja de gran interés desde el punto de vista del arreglo del telar. Mención aparte merecen los dos maravillosos volúmenes sobre diseños de textiles indígenas de todo México, registrando motivos de extraordinaria belleza que no habían sido documentados previamente, y que en muchos casos han caído en el olvido, por desgracia. Esa publicación generó tal interés entre el público que posteriormente se han editado dos selecciones distintas de los diseños en formato menor para amplia divulgación.

A lo largo de su carrera, la maestra Johnson asesoró a numerosos estudiantes. Prácticamente todos los investigadores más jóvenes del textil en México nos formamos con ella. Es proverbial la generosidad con que la maestra Johnson recibía a estudiantes, investigadores, y toda persona interesada en el textil en su casa en Coyoacán. Cabe hacer notar su asesoría de tesis para la maestra en etnolingüística Bartola Morales García (1987) en Ojitlán, que representa el primer trabajo de descripción e interpretación simbólica del textil por parte de una investigadora indígena.

Pasados sus ochenta años de edad, cuando la gran mayoría de los profesionistas se han retirado, la maestra Johnson continuaba activa en la investigación y publicación. Entre sus últimos trabajos están un registro de los textiles arqueológicos de todo el país, y un estudio del teñido mediante las técnicas de plangi y tritik, poco documentadas en México, en colaboración con la 
artista textil Virgina Davis (2002). Todavía en el 2004, al cumplir los noventa, publicó un análisis detallado de una variante poco conocida del tejido de gasa, una de las técnicas que evidencian el alto grado de sofisticación de las tejedoras mesoamericanas.

Quisiera resaltar, en conclusión, la importancia de la obra de la maestra Johnson desde una perspectiva humana. Irmgard no sólo fue una investigadora extraordinaria, en una época en que pocas mujeres participaban en la investigación, especialmente si involucraba las dificultades del trabajo de campo, cuando había que montar en bestia o caminar largos días para llegar a las comunidades campesinas. No sólo dedicó ella interminables horas al trabajo difícil y tedioso de analizar tejidos para contribuir a nuestro entendimiento de la historia material. Más que eso, la obra de Irmgard nos acerca a la humanidad de las tejedoras mexicanas: entre diagramas de hilos y descripciones minuciosas, nos hace admirar la habilidad de manos y la sutileza intelectual de las mujeres indígenas. El cariño y la entrega que conllevó ese acercamiento reflejan su propia grandeza de espíritu.

Alejandro de Ávila Blomberg Museo Textil de Oaxaca

\section{REFERENCIAS CITADAS}

Davis Virginia

2002 "Mexican stitch resist dyed and tie resist dyed textiles: a tradition vanishes », The Textile Museum Journal, 40-41, pp. 49-82.

EMERY Irene

1966 The primary structures of fabrics, The Textile Museum, Washington.

Morales García Bartola

1987 La elaboración de la indumentaria femenina chinanteca de Ojitlán, Oaxaca, tesis profesional, programa de etnolingüística, SEP/INI, Apetatitlán, Tlaxcala.

SEILER-BALdinger Annemarie

1994 Textiles: a classification of techniques, Smithsonian Institution Press, Washington.

\section{PrinCIPALES PUBLICACIONES DE IRMGARD WeITLANER JOHNSON}

1950 Twine-plaiting: a historical, technical, and comparative study, tesis de maestría, Universidad de California, Berkeley.

1952 Notas de campo, Universidad de Leiden [manuscrito inédito; www.rmv.nl/ publicaties/22Mixteeks_textiel/n/veldwerk_johnson.pdf]. 
1953 «El quechquemitl y el huipil», in Ignacio Bernal y Eusebio Dávalos H. (eds), «Huastecos, totonacos y sus vecinos», Revista Mexicana de Estudios Antropológicos, 13, pp. 241-257.

1954 "Chiptic Cave textiles from Chiapas», Journal de la Societé des Américanistes, 43, pp. 137-147.

1957a «An analysis of some textile fragments from Yagul », Mesoamerican Notes, 5, pp. 77-81.

1957b "Survival of feather ornamented huipiles in Chiapas, Mexico », Journal de la Société des Americanistes, nouvelle série, 16, pp. 189-196.

1962 con Jean B. Johnson y Grace C. Beardley, «Industrias y tejidos de Tuxpan, Jalisco, México », Anales del Instituto Nacional de Antropología e Historia, 14 (43), pp. 149-217.

1966 «Análisis textil del lienzo de Ocotepec », in Summa Anthropologica en homenaje a Roberto J. Weitlaner, INAH, México, pp. 139-144.

1967a «Un huipilli precolombino de Chilapa, Guerrero », Revista Mexicana de Estudios Antropológicos, 21, pp. 149-172.

1967b « Textiles », in Richard S. MacNeish, Antoinette Nelken-Terner y Irmgard W. Johnson, The prehistory of the Tehuacán valley. vol. 2. Nonceramic artifacts, University of Texas Press, Austin, pp. 189-226.

1967c «Miniature garments found in Mixteca Alta caves, Mexico », Folk, 8-9, pp. 179-190, Copenhague.

1971 «El vestido y el adorno », in Rafael Carrillo Azpeitia et al., Lo efímero y eterno del arte popular mexicano. 1, Fondo Editorial de la Plástica Mexicana, México.

1976a Design motifs on Mexican Indian textiles, Akademische Druck- und Verlagsanstalt, Graz.

1976 b «Weft-wrap openwork techniques in archaeological and contemporary textiles of Mexico », The Textile Museum Journal, 4 (3), pp. 63-72.

1977a Los textiles de la Cueva de la Candelaria, Coahuila, INAH, col. « Científica » 51, México.

1977b «Old-style wrap-around skirts woven by Zapotec Indians of Mitla, Oaxaca », in Irene Emery y Patricia Fiske (eds), Ethnographic textiles of the Western hemisphere. Irene Emery roundtable on museum textiles. 1976, proceedings, The Textile Museum, Washington, pp. 238-255.

1989 "The huisho and the quechquemitl in Santiago Temoaya, State of Mexico », in Yólotl González (ed.), Homenaje a Isabel Kelly, INAH, col. «Científica» 179, México, pp. 249-268.

1993 «Telas emplumadas en la época virreinal», in Teresa Castelló Yturbide et al., El arte plumaria en México, Fomento Cultural Banamex, México, pp. 79-99.

1996 «Anatomía de una tradición textil », in Ruth D. Lechuga (ed.), « Textiles de Oaxaca », Artes de México, 35, México, pp. 24-37.

2004 A rare type of Mexican gauze, Tetl, Leiden. 